\title{
The Effect of Citicoline on Electroretinography Abnormalities in Patients with Non-proliferative Diabetic Retinopathy
}

\author{
Anna Nur Utami ${ }^{1,3}$, Elvioza ${ }^{1,3}$, M. Sidik ${ }^{1,3}$, Aria Kekalih ${ }^{2,3}$ \\ ${ }^{1}$ Department of Ophthalmology, Universitas Indonesia, Depok, Indonesia \\ ${ }^{2}$ Department of Community, Universitas Indonesia, Depok, Indonesia \\ ${ }^{3}$ Cipto Mangunkusumo Hospital, Jakarta, Indonesia
}

\begin{abstract}
Introduction: to determine the effect of citicoline $1000 \mathrm{mg}$ oral supplementation given for 4 weeks on electroretinography abnormalities in patients with NPDR (non-proliferative diabetic retinopathy).

Methods : prospective, double blind, randomized clinical trial. Thirty-eight patients who matched the inclusion and exclusion criteria were randomized into two groups: the placebo (P-NPDR) and citicoline (C-NPDR). In the end, there were 18 eyes in citicoline group and 16 eyes in placebo group. The primary outcome was $P 50$ and N95 amplitude in PERG within group and intergroup which were taken at the baseline and 4 weeks after treatment.
\end{abstract}

Results : at the end of treatment, the N95 amplitude in C-NPDR showed improvement, 4.85 (1.9-10.3) $\mu \mathrm{V}$, before treatment to 5.7 (1.9-17.1) $\mu \mathrm{V}$, after treatment with $P=0.04$. Median $P 50$ amplitude improved in both groups, with C-NPDR: $3.1 \mu \mathrm{V}$ to $3.8 \mu \mathrm{V}(p=0.89)$, and P-NPDR: $3.5 \mu \mathrm{V}$, to $4.5 \mu \mathrm{V}(p=0.10)$. Delta $\triangle N 95$ amplitude is higher in C-NPDR, while delta $\triangle P 50$ amplitude is higher in P-NPDR, with P values 0.35 and 0.45 .

Conclusion : Oral citicoline may induce a significant improvement in mean $N 95$ amplitude before and after the treatment. P-NPDR showed positive trend in P50 amplitude while in C-NPDR showed positive trend in N95 amplitude, but these values were not statistically significant $(P=0.45 ; P=0.35)$.

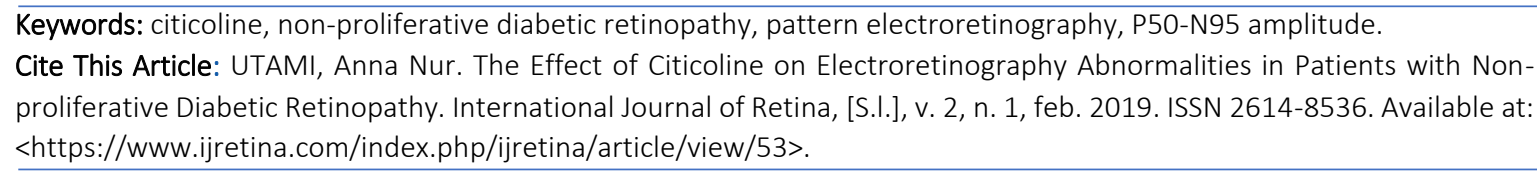

\section{INTRODUCTION}

Diabetes mellitus (DM) is a metabolic disorders caused by hyperglicemia. ${ }^{1}$ In 2000 , at least 172 million people or $2.8 \%$ of world population had diabetes. Diabetic retinopathy is one of microvascular complication of DM. Approximately, $25-50 \%$ of type 1 diabetes patients have retinopathy after 10 years, while in type 2 patients, 23\% will have retinopathy. WHO reported that diabetic retinopathy is responsible for $4.8 \%$ of the 37 million cases of blindness in the world. ${ }^{2}$

The retinal changes in diabetic retinopathy are a consequence of a damage in retinal microvasculature. The retinal cells involved in diabetic retinopathy are endothelial cells and neuronal cells. Studies around these days showed that significant neuronal damage is an earlier event that vascular damage. ${ }^{3}$ Diabetes can caused neural apoptosis by several mechanisms, include: ischemia, oxidative stress, glutamate excitotoxity, neuroinflamation, and increased activity of aldose reducatse. ${ }^{4}$ Ganglion cells, in the inner layer of the retina, are the most involved cells in the pathological process in diabetes. ${ }^{5}$

Citidine diphosphocoline (CDP-choline or citicoline) CDP-choline is an essential intermediate in the synthesis of structural phospholipids of cell membranes, phosphatidylcholine. $^{6}$ After neural death process, exogen citicoline will participate in the phospholipid synthesis at the neuronal membrane cells. Several studies also reported that citicoline also have neuroprotective effect in retinal ganglion cells and promotes nerve regeneration in vitro. ${ }^{7}$ 
Electroretinography examination is an electric functional examination that aims to evaluate the retina function. Pattern electroretinography (PERG) is a retinal biopotential evoked by a patterned checkerboard stimulus. There are two components in PERG, which are P50 and N95 amplitudes. PERG can provide information about the macular and retinal ganglion cell function. ${ }^{8}$ These retinal ganglion cells are influenced by oral citicoline.

The management of diabetic retinopathy in the early phase is limited by risk factors controlled, such as hiperglycemia, hypertension, and dyslipidemia. While in diabetes mellitus, the ischemia caused by the gyperglicemia activates a number of proinflammatory pathways, and cause a long-lasting persistent effect even after the blood glucose is normal (hyperglicemic memory). ${ }^{9}$ Thus, the aim of this study is to evaluate the effect of oral citicoline on electroretinography in patients with NPDR.

\section{METHODS}

This study was a prospective, double blind, randomized clinical trial study, and held in Vitreoretina and Neuroophthalmology division Kirana- Cipto Mangkunkusumo hospital from March to July 2016. Ethical approval and informed consent were obtained.

The inclusion criteria was patients in moderate / severe NPDR which has not been treated by PRP, aged 20-65 years old, and HBA $1 \mathrm{C}<8 \%$. Patients will be excluded if the bestcorrected visual acuity $<20 / 50$, significant media opacity (moderate - severe), macular / optic nerve disease other than diabetic retinopathy, and previous antioxidant / neuroprotective treatment within 2 weeks. Patient with worsen progressitivity of the disease who needs the PRP treatment, having a protocol violation, or loss to follow up will be dropped out from this study.

Consecutive sampling was done and subjects were randomized (block randomization) into 2 groups: placebo and citicoline. Only one eye which met the criteria included in the study. Subject who met inclusion and exclusion criteria underwent BCA examination using EDTRS chart, funduscopy with 78D lens, fundus photograph, and ERG examination (PERG and full-field ERG). All electrophysiological examinations were performed using an ISCEV standard. The subjects under examination were seated in an acoustically isolated semi-dark room in front of a display surrounded by a uniform field $(120 \cdot 120$ degree) of luminance $(5 \mathrm{~cd} / \mathrm{m} 2)$.
The visual stimuli were checkerboard patterns (contrast was $93 \%$, mean luminance $100 \mathrm{~cd} / \mathrm{m} 2$ ) generated on a television monitor and reversed in contrast at the rate of 4 reversals per second. The electrode used was DTL-plus. The pharmacologic treatment was given for 4 weeks in a daily intraoral dose of $1000 \mathrm{mg}$ citicoline or placebo.

The primary outcome in this study was P50 amplitude and N95 amplitude, with BCVA as a secondary outcome. Evaluation was done in 4 weeks post intervention. The side effect of the drugs was also recorded,

Statistical analysis was performed using SPSS 21.0. The numeric data is analyzed with $t$ test or Mann- Whitney test. Paired t-test was used to compare mean amplitude P50 and N95 amplitude within group.

\section{RESULT}

There were 38 eyes included in the beginning of the study. 4 subjects were dropped out because of 2 subjects had gastrointestinal discomfort and 2 subjects were loss to follow up. Thirty-four eyes were analyzed, with 18 eyes in citicoline group and 16 eyes in placebo groups. Table 1 shows the baseline characteristic of patients in the study include: age, gender, HbA1c, duration of DM, dyslipidemia, hypertension, and degree of retinopathy with or without macular edema. Baseline characteristics of the subjects in both groups were similar $(p>0.05)$.

Table 2 shows the baseline data before the intervention in two groups; include P50 amplitude, N95 amplitude, and BCVA. The statistical analysis from the two groups were $p>0.05$, which can be concluded that all data from both groups were similar.

The P50 and N95 amplitude was assessed at day-0 (baseline) and 4 weeks post intervention. Clinically, the result in citicoline groups showed improvement in all variables. Median P50 amplitude before treatment, $3.1 \mu \mathrm{V}$, increased to $3.8 \mu \mathrm{V}(p=0.89)$, median $\mathrm{N} 95$ amplitude increased from $4.85 \mu \mathrm{V}$ to $5.7 \mu \mathrm{V}(p=0.04)$, and median BCVA LogMar improved from 0.13 LogMar to 0.10 LogMar $(p=0.02)$. In placebo group, only P50 amplitude improved from $3.5 \mu \mathrm{V}$ to $4.5 \mu \mathrm{V}(p=0.10)$. Figure 1 and 2 show the comparison in P50 and N95 amplitude post intervention between two groups. 
Table 3 exhibited a comparison in delta examination after the intervention in both groups. Delta examination results were obtained from the amplitude difference between the pre and post intervention. Delta N95 amplitude was higher in citcioline group ( $p=0.35)$ while the delta P50 amplitude was higher in the placebo group ( $p=0.45$ ). Total subjects in this study were 38 subjects. There were four-drop outs. After the randomization table was openned, we found that these subjects were from placebo groups.

Table 1. Baseline Characteristics

\begin{tabular}{|c|c|c|c|c|}
\hline & Variable & Placebo & Citicoline & $\mathbf{p}$ \\
\hline Subjects (n) & & 16 & 18 & \\
\hline Age (years) & & $55.56 \pm 7.85^{*}$ & $56.83 \pm 6.93^{*}$ & $0.6^{\mathrm{a}}$ \\
\hline \multicolumn{5}{|l|}{ Gender $(\%)$} \\
\hline$\bullet$ & Female & $9(56.2 \%)$ & $10(55.6 \%)$ & $0.97^{\mathrm{b}}$ \\
\hline$\bullet$ & Male & $7(43.7 \%)$ & $8(44.4 \%)$ & \\
\hline \multicolumn{5}{|c|}{ Duration DM (years) } \\
\hline$\bullet$ & $<10$ years & $7(43.7 \%)$ & $10(55.6 \%)$ & $0.49^{b}$ \\
\hline$\bullet$ & $>10$ years & $9(56.2 \%)$ & $8(44.4 \%)$ & \\
\hline HbA1c $(\%)$ & & $7.5(4-8.1)^{* *}$ & $7(6-8) * *$ & $0.76^{\mathrm{c}}$ \\
\hline \multicolumn{5}{|l|}{ Hipertension } \\
\hline$\bullet$ & Yes & $2(12.5 \%)$ & $7(38.9 \%)$ & $0.08^{\mathrm{d}}$ \\
\hline$\bullet$ & No & $14(87.5 \%)$ & $11(61.1 \%)$ & \\
\hline \multicolumn{5}{|l|}{ Dyslipidemia } \\
\hline$\bullet$ & Yes & $5(31.2 \%)$ & $7(38.9 \%)$ & $0.64^{b}$ \\
\hline$\bullet$ & No & $11(68.8 \%)$ & $11(61.1 \%)$ & \\
\hline \multicolumn{5}{|l|}{ Diagnosis } \\
\hline$\bullet$ & Moderate NPDR & $9(56.2 \%)$ & $14(77.8 \%)$ & $0.27^{b}$ \\
\hline$\bullet$ & Severe NPDR & $7(43.8 \%)$ & $4(22.2 \%)$ & \\
\hline$\bullet$ & With macular edema & $7(43.8 \%)$ & $7(38.8 \%)$ & $0.77^{b}$ \\
\hline $\begin{array}{c}\bullet \\
\text { edema }\end{array}$ & Without macular & $9(56.2 \%)$ & $11(61.1 \%)$ & \\
\hline
\end{tabular}

a: T-test; b: Chi square; c: Mann-Whitney test; d: Fisher test, *: mean \pm SD, ${ }^{* *}$ : median (min-max)

Table 2. Baseline data before the intervention in two groups

\begin{tabular}{lccc}
\hline \multicolumn{1}{c}{ Variable } & placebo & Citicoline & p \\
\hline Amplitude P50 $(\mu \mathrm{V})$ & $3.6 \pm 1.5^{*}$ & $3.8 \pm 2.9^{*}$ & $0.80^{\mathrm{a}}$ \\
Amplitude N95 $(\mu \mathrm{V})$ & $4.4 \pm 1.9^{*}$ & $5.3 \pm 2.5^{*}$ & $0.28^{\mathrm{a}}$ \\
BCVA (LogMar) & $0.21 \pm 0.16^{*}$ & $0.17 \pm 0.16^{*}$ & $0.29^{\mathrm{a}}$ \\
\hline
\end{tabular}

BCVA : best corrected visual acuity; a: t-test ; *: mean $\pm \mathrm{SD}$;

Table 3. Comparison of delta $\Delta$ evalution post-intervention between the two groups

\begin{tabular}{cccc}
\hline Variable & Placebo & Citicoline & p \\
\hline$\Delta$ amplitude P50 $(\mu \mathrm{V})$ & $0.90 \pm 2.1^{*}$ & $0.21 \pm 3.1^{*}$ & $0.45^{\mathrm{a}}$ \\
$\Delta$ amplitude N95 $(\mu \mathrm{V})$ & $0.03 \pm 2.4^{*}$ & $0.81 \pm 2.3^{*}$ & $0.35^{\mathrm{a}}$ \\
$\Delta$ BCVA (LogMar) & $0.0(-0.10-0.42)^{* *}$ & $0.0(-0.18-0.30)^{* *}$ & $0.08^{\mathrm{b}}$ \\
\hline
\end{tabular}

a: T-test; b: Mann-Whitney test *: mean $\pm \mathrm{SD} ; * *$ : median (min-max) 


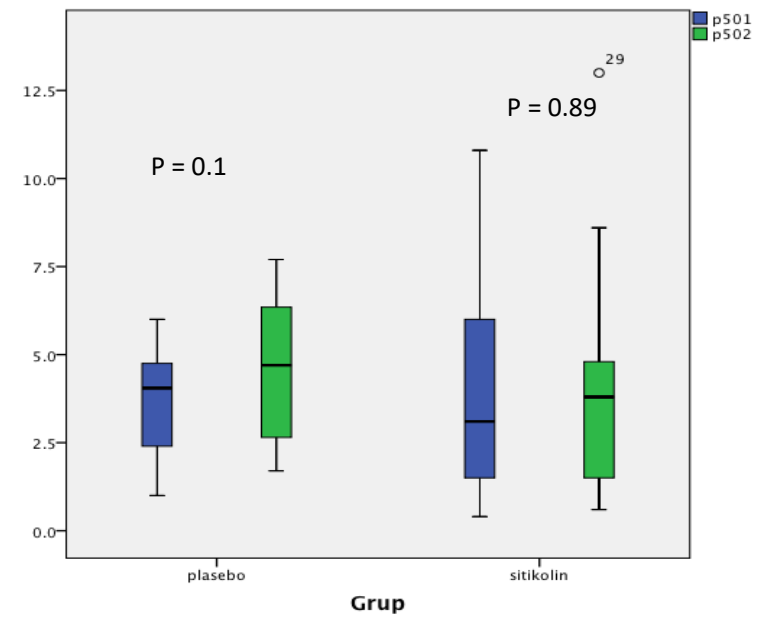

Figure 1. Comparison of P50 amplitude pre- and postintervention between the two groups

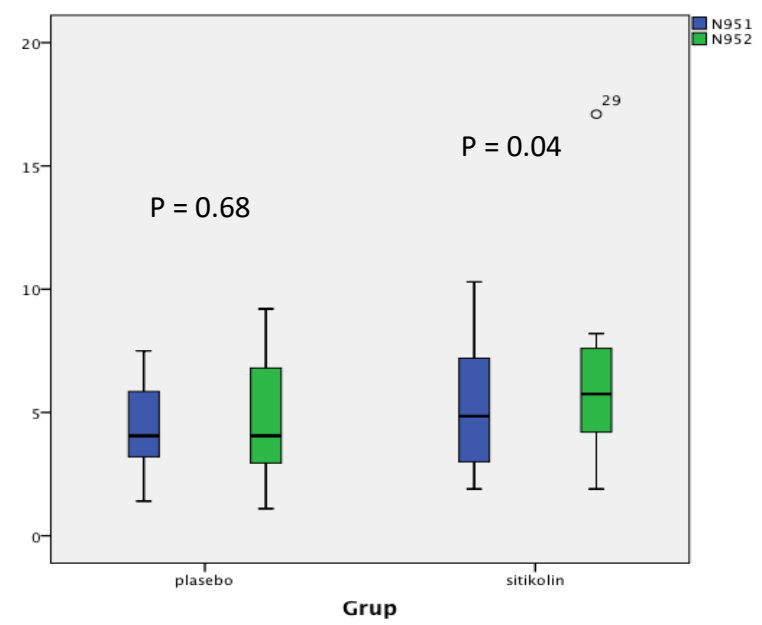

Figure 2. Comparison of N95 amplitude pre- and postintervention between the two groups

\section{DISCUSSION}

Variables included in the baseline characteristics were gender, age, HbaA1c, duration of DM, hypertension, dyslipidemia, and degree of retinopathy. The proportion of the gender in this study is almost equal between the female and male, with percentages of $56 \%$ female and $44 \%$ male. Similar data was reported by Yau et al ${ }^{10}$, that the prevalence of diabetic retinopathy in women $(52 \%)$ and men $(48 \%)$ are the same. The mean age of the subjects in this study was $56.24 \pm 1.25$ years old. This characteristics was similar to epidemiological studies conducted in 22.896 subjects of American, Australian, European, and Asian, with mean age of 58.1 years old. ${ }^{10}$

This study included only subjects with $\mathrm{HbA} 1 \mathrm{c}$ levels $<8 \%$. David $M D$, et al ${ }^{11}$ reported that the risk of progression in diabetic retinopathy is associated with $\mathrm{HbA} 1 \mathrm{c}$ levels in one's blood. Study from Raman ${ }^{12}$ also reported that the levels of $\mathrm{HbA} 1 \mathrm{c}>8 \%$ was significantly associated with the incidence of sight-threatening diabetic retinopathy (severe NPDR, proliferative diabetic retinopathy and clinically significant macular edema). Diabetic retinopathy is a multifactorial microvascular complications associated with hyperglycemia, hypertension, and high lipid levels in the blood. The proportion of subjects with hypertension in this study was $26.5 \%$. Wisconsin study also reported that the prevalence of hypertension in type 1 diabetes was $17.3 \%$ and $25.9 \%$ after 10 years of diabetes. ${ }^{13}$ Chew et al ${ }^{14}$ in the Early Treatment Diabetic Retinopathy Study (EDTRS) reported patients with elevated levels of total cholesterols and high LDL may exacerbate the occurance of retinopathy and the formation of hard exudates.

One of the purposes of this study was to evaluate the results of the examination before and after the intervention within group. P50 amplitude was clinically improved in both groups before and after treatment, but these values were not statistically significant ( $p>0.05$ ). P50 amplitude was increased higher in the placebo group. The P50 component is more specific to be an indicator of macular function. ${ }^{15}$ Improvement in the P50 amplitude could be caused by improvement of subject's risk factors, such as hypertension and dylipidemia that lead to reduction in hard exudates and intraretinal hemorrhages in the macula. All subjects in this study were consulted to the internist at the first visit. Idiculla et $\mathrm{al}^{16}$ reported that serum lipids has a significant connection with the formation of hard exudates, clinically significant macular edema (CSME), and loss of visual acuity in type 2 diabetes. Study by Rusenberg ${ }^{17}$ reported that diabetes could cause swelling and beading on the dendrites and axons of retinal ganglion cells.

N95 in PERG is a component that reflects the retinal ganglion cell layer. Several studies reported that N95 amplitude is directly related to the volume of retinal ganglion cells. Exogenous citicoline can increase the formation of phosphatidylcholine (ptdCho) and phospholipids, stabilizes the intracellular conditions of neuronal cells, decrease the activity of phospholipase A2 enzyme (PLA2), and antipoptosis in mitochondria pathway. ${ }^{18-23}$ Figure 2 shows that pre- and postintervention N95 amplitude only inrease in citicoline group. There was an increase in the median of N95 amplitude before treatment, $4.85 \mu \mathrm{V}$, to $5.7 \mu \mathrm{V}$ after treatment, which statistically significant $(p=0.04)$ in citicoline group. Previous study regarding the used of citicoline in glaucoma and NAION also reported similar ideas. Parisi2 ${ }^{24}$ reported that the administration of intramuscular citicoline in $1000 \mathrm{mg} /$ day for 60 days in patients with glaucoma might improve the visual function as suggested by the increase in P50-N95 amplitude and N75-P100 VEP. Parisi ${ }^{25}$ also had research on citicoline administration in patients with NAION. In his research, citicoline given in $1600 \mathrm{mg} / \mathrm{diem}$ for 60 day 
improved the PERG and VEP in patients. The secondary outcome of this research is BCVA variable. The BCVA improved by 0.03 LogMar in citicoline group, with $p$ value of 0.02 . This finding is consistent with previous study that also reported that citicoline can improve visual acuity. Citicoline is a neuroprotective agent, which plays an important role in the synthesis of phospholipid in cell membrane. Additional functions of phospholipid in neuronal membrane is to impulse conduction of nerve cells and neurotransmitter. ${ }^{6}$

Another objective of this study was to look at the differences in test results pre- and post-intervention in both groups (intergroup). Table 3 shows that the N95 amplitude in citicoline group had better improvement that the placebo group, but unfortunately from the statistically analysis, the $p$ value was $>0.05$. This value could be explained by several reasons, such as: distribution value was abnormal in both groups, which made the standard deviation was quite large. Standard deviation entered in the calculation of the sample in this study was $0.7 \mu \mathrm{V}$, while the standard deviation obtained from the results is $1.9 \mu \mathrm{V}$. Wide variation in this study is probably because of differences in visual acuity. severity of retinopathy, and large variations in duration of DM.

Total subject in this research is 38 . Only 2 subjects had gastroinstetinal complaint after the intervention. These two subjects were from placebo group. There was zero person from the citicoline group. This result is similar to other studies that reported citicoline are safe and the side effects that are attributable to this drug are rare. The side effect consist mainly of headache and gastrointestinal discomfort (5.01\%). ${ }^{6}$

\section{CONCLUSION}

Daily dose $1000 \mathrm{mg}$ of citicoline given in 4 weeks may induce a significant improvement in mean N95 amplitude before and after the treatment. In placebo group, there was a positive trend in P50 amplitude while in citicoline group showed positive trend in N95 amplitude, but these values were not statistically significant.

\section{REFERENCES}

1. Miranda M, Sanches MV, Alvares R, Vilela C, Romero FJ. Electroretinogram alteration in diabetes. Electroretinograms. Dr. Gregor Belusic (ed.) InTech; 2011. [cited 2015 December]. Available from: http://intechopen.com.

2. Prevention of blindness from diabetes mellitus: report of a WHO consultation in Geneva [internet]. Switzerland: World Health Organization; 2005 Nov [cited 2015 Des]. 7p. Available from: http://who.int.
3. Matteucci A, dkk. Primary retinal cultures as a tool for modeling diabetic retinopathy: an overview. Hindawi. Biomed research international. 2015.

4. M. Seki, T. Tanaka, H. Nawa et al. Involvement of brain- derived neurotrophic factor in early retinal neuropathy of streptozotocin-induced diabetes in rats: therapeutic potential of brain-derived neurotrophic factor for dopaminergic amacrine cells. Diabetes. 2004;53:241219.

5. Bikbova G, Oshitari T, Yamamoto S. Diabetes Mellitus and Retinal Vein Occlusion as Risk Factors for Open Angle Glaucoma and Neuroprotective Therapies for Retinal Ganglion Cell Neuropathy. J Clinic Experiment Ophthalmol. 2012. S3:002.

6. Secades JJ. Citicoline: pharmacological and clinical review, 2010 update. Rev Neurol 2011; 52 (Suppl 2): S1-62.

7. Oshitari T, Fujimoto N, Adachi-Usami E. Citicoline has a protective effect on damaged retinal ganglion cells in mouse culture ret- ina. NeuroReport 2002; 13: 2109-11.

8. Bach $M$, Hoffmann $M$. Update on the pattern electroretinogram in glaucoma. Optom Vis Sci. 2008;85:386-95.

9. Giacco F, Brownlee M. Oxidative stress and diabetic complications: Circ Res.2010;107(9):1058-70.

10. Yau dkk. Global prevalence and major risk factors of diabetic retinopathy. Diabetes Care. 2012; 35(3): 556-64.

11. Davis $M$, Fisher $M$, Gangnon $R$, et al. Risk Factors for High-Risk Proliferative Diabetic Retinopathy and Severe Visual Loss: Early Treatment Diabetic Retinopathy Study Report \#18. Invest Ophthalmol Vis Sci.1998;39:233-52.

12. Raman R, Verma A, Pal S, Gupta A, Vaitheeswaran $K$, Sharma T. Influence of glycosylated hemoglobin on sight-threatening diabetic retinopathy:A population-based study. Diab Res Clin Pract. 2011:1-6.

13. Klein R, Knudtson MD, Lee KE, Gangnon R, Klein BE. The Wisconsin epidemiologic study of diabetic retinopathy: XXII the twent-five-year progression of retinopathy in persons with type 1 diabetes. Ophthalmology. 2008; 115(11):1859-68.

14. Chew dkk. Association of elevated serum lipid levels with retinal hard exudate in diabetic retinopathy. Early Treatment Diabetic Retinopathy Study (ETDRS) Report 22. Arch Ophthalmol. 1996 Sep; 114(9):1079-84.

15. Kreuz AC, Oyamada MK, Hatanaka M, Montero ML. The role of pattern-reversal electroretinography in the diagnosis of glaucoma. Arq Biras Oftalmol. 2014;77(6):40310.

16. Idiculla J, Nithyanandam S, Joseph M, Ajoy Mohan VK, Vasu U, Sadiq M. Serum lipids and diabetic retinopathy: A cross-sectional study. Indian J Endocrinol Metabl. 2012. 16(2): S492-4. 
17. Rusenberg BM, Pavlidids $\mathrm{M}$, Stupp $\mathrm{T}$, Thanos $\mathrm{S}$. Pathological changes in human retinal ganglion cells associated with diabetic and hypertensive retinopathy. Graefe's Archive for Clinical and Experimental Ophthalmology. 2006. 245(7): 1009-18.

18. Grieb P, Redjak R. Pharmacodynamics of citicoline relevant to the treatment of glaucoma. Journal of Neuroscience Research. 2002;67:143-8.

19. Seki $M$, Tanaka $T$, Nawa $H$, et al. Involvement of brain-derived neurotrophic factor in early retinal neuropathy of streptozotocin- induced diabetes in rats: therapeutic potential of brain-derived neu- rotrophic factor for dopaminergic amacrine cells. Diabetes 2004; 53: 241219.

20. Siesjo B. K. Pathophysiology and treatment of focal cerebral ischemia II: mechanisms of damage and treatment. J. Neurosurg. 1992;77:337-54.

21. Park $\mathrm{CH}$, Kim YS, Lee HK, Kim YH, Choi MY. Citicoline reduces upregulated clusterin following kainic acid injection in the rat retina. Current Eye Research. 2007;32:1055-106.

22. Alberghina M, Giuffrida-Stella AM. Changes of phospholipid- metabolizing and lysosomal enzymes in hypoglossal nucleus and ventral horn motoneurons during regeneration of craniospinal nerves. J Neurochem. 1988;51:15-20.

23. Lynch MA, Voss KL. Arachidonic acid increases inositol phospholipid metabolism and glutamate release in synaptosomes prepared from hippocampal tissue. J Neurochem. 1990; 55: 215-21.

24. Parisi V. Electrophysiological assesment of glaucomatous visual dysfunctiin during treatment with cytidine-5-diphosphocholine (citicoline): a study of 8 years of follow-up. Documenta Ophthalmologica. 2005;110: 91102.

25. Parisi V, Coppola G, Ziccardi L, Gallinaro G, Falsini B. Cytidine 5-diphosphocoline (citicoline): a pilot study in patients with non-arteritic ischemic optic neuropathy. European Journal of Neurology 2008,15: 465-474.

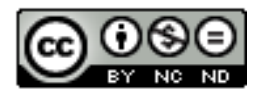

This work licensed under Creative Commons Attribution 